\title{
Visualizing Transaction Context in Trust and Reputation Systems
}

\author{
Johannes Sänger \\ Department of Information Systems \\ University of Regensburg \\ Regensburg, Germany \\ Johannes.Saenger@wiwi.uni-regensburg.de
}

\author{
Günther Pernul \\ Department of Information Systems \\ University of Regensburg \\ Regensburg, Germany \\ Guenther.Pernul@wiwi.uni-regensburg.de
}

\begin{abstract}
Transaction context is an important aspect that should be taken into account for reputation-based trust assessment, because referrals are bound to the situation-specific context in which they were created. The non-consideration of transaction context may cause several threats such as the value imbalance problem. Exploiting this weakness, a seller can build high reputation by selling cheap products while cheating on the expensive ones. In the recent years, multiple approaches have been introduced that address this challenge. All of them chose metrics leading to numerical reputation values. These values, however, are non-transparent and quite hard to understand for the end-user. In this work, in contrast, we combine reputation assessment and visual analytics to provide an interactive visualization of multivariate reputation data. We thereby allow the user to analyze the data sets and draw conclusions by himself. In this way, we enhance transparency, involve the user in the evaluation process and as a consequence increase the users' trust in the reputation system.
\end{abstract}

Keywords-trust, reputation, context, transaction context, context-awareness, visual analytics, visualization, parallel coordinates

\section{INTRODUCTION}

The notion of trust has been widely discussed in the recent decades. It has been identified to be a key factor for success in many eEnvironments such as eCommerce or peer-to-peer networks [1]. Unlike traditional face-to-face transactions, electronic transactions are carried out between parties that have not physically met. Thus, transaction partners are usually strangers whose trustworthiness is unknown. Particularly in online markets, where advance payment is a common practice, buyers face high risks. To reduce the uncertainty, reputation systems have established which allow to rate actors based on past experiences made.

Although neglected in the trust research community for a long time, transaction context has been recognized to play an important role for trust assessment today [2]. In eCommerce, for instance, transactions are accomplished involving different prices, product types, payment methods, quality or time. The non-consideration of the price may lead to the "value imbalance problem" where a malicious seller can build a high reputation by selling cheap products while cheating on expensive ones [3]. Zhang et al. [4] extended this phrase to cover the whole transaction context when speaking of the "transaction context imbalance problem". In the recent years, many trust models and metrics were proposed to address context in trust assessment. However, all of them apply a purely numerical approach in which the trustworthiness of an actor in a specific situation is represented by one or several final reputation values. Since the metric behind the values as well as the reputation data that served as input for the reputation assessment is not explicitly shown, these approaches seem quite non-transparent and inexplicable to the user, as Hammer et al. [5] could show.

In this work, we are taking a step to enhance transparency in trust and reputation systems by developing a visual representation of a context-sensitive reputation profile. We apply visual analytics which combines the strength of both a computer and a human analyst to improve reasoning and decision making based on complex and multivariate data sets. Various interactions techniques support the user in revealing valuable insights to gain knowledge by visually exploring the datasets. In this way, we want to involve the user in the evaluation process and as a consequence increase the users' trust in the reputation system.

The remainder of this paper is based on the design science research paradigm including the guidelines for conducting design science research by Hevner et al. [6]. In particular, we follow the "nominal process model for the conduct of design science" introduced by Peffers et al. [7] which is based on the guidelines provided by Hevner et al. We firstly give an overview of the problem context and related work in Section II. Based on this, we clarify our motivation, identify the research gap and define the objectives of our work. Secondly, we propose our novel visualization-based approach for reputation systems in Section III. Thereby, we elaborate a conceptual design for visual analytics of reputation data that is independent of the application area. We then demonstrate how this conceptual design can be prototypically implemented using the example of an eCommerce dataset from eBay. To give an impression of how the implemented prototype proves itself in practice, we subsequently conduct an empirical study where two cases are introduced (Section IV). In Section V, we point out our contribution and discuss further challenges. We thereby name several questions which will be focal points for our 
future research. Finally, we sum up our work and conclude in the last Section VI.

\section{Problem Context And Related Work}

It has been widely recognized that context is an important aspect that should be taken into account for trust evaluation, because referrals are bound to the specific context in which they were created. To impart a common understanding of the notions of trust, reputation and context, we first provide a short description and definition of these terms. Then, we give an overview of the main research areas inspiring our work. Thereby, related concepts regarding context in trust and reputation systems are shortly expounded. At the same time, visual analytics and the visual analytics process pipeline will be introduced. Based on this, we point out the research gap and define the objectives of this work.

\section{A. Trust, reputation and transaction context}

The notion of trust has been discussed by researchers for decades. However, it still lacks in a uniform and commonly agreed definition. This is mostly due to the fact that trust is a multifaceted concept related to various terms like credibility, reliability or confidence. It has a cognitive, an emotional and a behavioral dimension. As pointed out by [8], trust has been described as an interpersonal phenomenon by psychologists while sociologists viewed trust as being structural in nature. Economists, however, interpreted trust as rational choice mechanism. The definition mostly cited in research regarding online trust and reputation that is referred to as reliability trust was proposed by Gambetta in 1988 [9]: "Trust (or, symmetrically, distrust) is a particular level of the subjective probability with which an agent assesses that another agent or group of agents will perform a particular action, both before he can monitor such action (or independently of his capacity ever to be able to monitor it) and in a context in which it affects his own action".

Multiple authors furthermore include security and risk which can lead to more complex definitions. Falcone and Castelfranchi for example point out that high reliability may not be sufficient for a positive decision. This becomes obvious, if the consequences of failure are too serious for a transaction to be taken into consideration [10]. To establish trust, a wide range of trust models has been proposed in the recent years. Thereby, two common approaches can be distinguished, namely policy-based and reputation-based trust establishment [11]. Policy-based trust relies on the handover of credentials such as a password, whereas reputation-based trust is based on the experiences made in past transactions. This history of interactions can be seen as an estimation of trustworthiness. Reputation is thereby defined as follows: "Reputation is what is generally said or believed about a person's or thing's character or standing” [12].

Besides information that is explicitly created for trust assessment such as user ratings, implicit information like the position of a node in a social network is often additionally examined. In this work we focus on reputation-based trust. Context as the third term important with regard to this work involves all the circumstances and situation specific attributes which can affect an action. Since context is also an issue that plays a role in several research fields, definitions are miscellaneous. In this work, we refer to the definition of transaction context proposed by Dey [13]: "Context is any information that can be used to characterize the situation of an entity. An entity is a person, place, or object that is considered relevant to the interaction between a user and an application, including the user and applications themselves".

Context-aware computing was first discussed by Theimer and Schilit [14]. Today, context-awareness has been recognized to be an important issue in many areas of research, such as recommender systems [15] or service provision [16]. In this work, we focus on context in trust and reputation systems.

\section{B. Context in Trust and Reputation Systems}

While context has been neglected by the trust research community for a long time, several authors proposed trust and reputation metrics considering context in the recent years. REGRET, a reputation model introduced in year 2001, already included an ontological dimension where different attributes could be modeled [17]. Although not explicitly talking of transaction context, this can be seen as one of the early approaches to include context in the reasoning process. Younger models such as CAT [18] or RateWeb [19] allow to model multidimensional context attributes such as delivery time or product quality. Other authors, like Rehak et al. [2], focused on referral context by providing an universal mechanism that allows to extend existing trust models by the capabilities to efficiently modeling situational trust. Zhang et al. [4] furthermore developed a trust vector approach where the single context dimensions are depicted by a numerical trust vector.

All of these works have one essential thing in common: Trust and reputation values are represented in one or several final numerical values. Many approaches apply similarity metrics to measure the distance of two referrals in a context space. However, these values are highly non-transparent since it does not become clear to the end-user how they were calculated. In this work, in contrast, we aim to allow a visual exploration of reputation by means of a graphical presentation of referrals and their context attributes. We think that we can only reach total transparency if the user can easily evaluate referral data by himself. We therefore make use of visual analytics.

\section{Visual Analytics}

Visual analytics (VA) is an interdisciplinary and fastgrowing field of research that combines automated analysis 
techniques with interactive visualizations. Incorporating the visual-cognitive capabilities of a human analyst, an effective understanding, reasoning and decision making on basis of very large and complex datasets is achieved [20]. Figure 1 shows an abstract overview of the VA process pipeline.

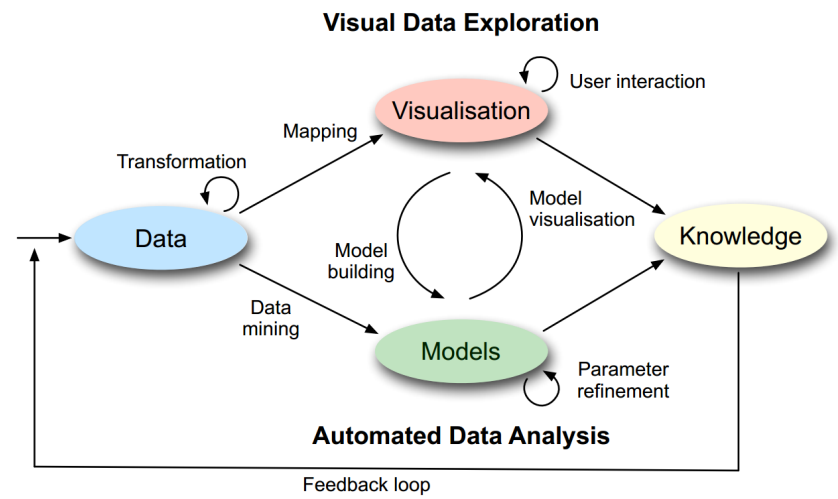

Figure 1. "The visual analytics process is characterised through interaction between data, visualisations, models about the data, and the users in order to discover knowledge" [20]

Starting point of the VA process are the raw data. These are transformed and integrated for further use first. Then, they serve as input for automated data analyses where models of the data are created. These models are visualized and provided to the analyst in an interactive visualization. User interaction finally allows to reveal insightful information and generate knowledge, for instance by zooming into different parts of the visualization or filtering unimportant information. Here, the user follows the maxim "detect the expected and discover the unexpected" [21].

VA has not yet been applied in reputation systems to the best of our knowledge. Examples of trust and reputation systems including visualizations mostly create static relationship graphs of the trust networks such as [22]. Thus, user interaction is a new feature.

\section{Research Gap: Visualization of Transaction Context}

Most metrics used in common reputation systems are quite non-transparent, since they only provide an aggregated reputation value that does not reveal details of how it came about. Hammer et al. [5] conducted a user-centric study of reputation metrics in which they could show that more than half of the participants criticized the lack of transparency. Improved transparency could therefore notably enhance users' experience in reputation systems [5]. Visualizations are perfectly suitable for easily depicting correlations between single attributes. The fact that all input data could be depicted in one visualization is a further advantage of visual representations. However, there is currently no approach that tries to apply visual analytics to evaluate referrals in reputation systems. In this work, we are taking the first step to combine visual analytics and reputation systems.
We provide a generic mechanism to model context-specific reputation by means of an interactive visualization. We, then, demonstrate how this mechanism can be implemented by the example of an eCommerce setting. Thus, the objectives of our solution are as follows:

- We want to enhance transparency by depicting all input data in one integrated view.

- We want to involve the users in the reputation assessment process to the end that the users' trust in the reputation system and the derived reputation values is increased.

\section{NOVEL VISUALIZATION-BASED APPROACH}

In this section, we introduce our novel visualizationbased approach to context-dependent reputation evaluation. Adapting the visual analytics process, we firstly describe the two building blocks "models" and "visualization \& interaction techniques". We delineate a conceptual design for each building block which is independent of the raw data provided for reputation assessment. Thus, this concept serve as a generic mechanism that can be adapted to a specific application area. Subsequently, we show how the conceptual design can be implemented by the example of an eCommerce dataset from eBay.

\section{A. Conceptual design}

1) Building block - models: Reputation is contextdependent since referrals are created in a particular situation bound to a transaction specific context. Therefore, a single referral that might be provided in the form of a uni-variate rating (e.g. $r=\{-1,0,1\}$ ) only reflects an opinion conceived in a specific situation. However, the context information gets lost in this representation not including any further information. To create a more comprehensive view, we model each referral in a multidimensional referral space $S$, where the rating is extended by multiple context attributes. Each dimension of the n-dimensional space $S$ corresponds to a relevant attribute (e.g. rating, time, product). The definition of the referral space $S$ is carried out in two steps:

1) We identify all relevant context attributes that represent the situation a referral was created. These attributes form our context set $C=\left\{c_{1}, c_{2}, \ldots c_{n}\right\}$, where each element $c_{i}$ takes values of the related set $C_{i}$. The value range of $C_{i}$ depends on the type and structure of the context attributes and can be continuous or discrete intervals as well as values of a nominal set. Exemplary continuous intervals are time or price, whereas size of market basket or user rating depict examples for discrete intervals. Manufacturer or product type are represented in a nominal set.

2) We define an n-dimensional referral space $S$ where each dimension matches a relevant context attribute (one dimension depicts the rating):

$$
S:=C_{1} \times C_{2} \times \ldots \times C_{n}
$$


Once the referrals space $S$ is defined, each referral denotes one point in the n-dimensional space. From here, we take a different path compared to other approaches. Instead of measuring the distance of two points to derive their similarity, we map the referral space $S$ to a 2-dimensional visualization space in the second step. In this way, we desist from an automatic numerical evaluation and let the user perform the analysis. Here, a combination of a visual and a numerical evaluation is also thinkable. To carry out the mapping, a convenient visualization technique needs to be selected.

2) Building block - Visualization and interaction techniques: There is wide range of visualization techniques suitable for multidimensional data sets. Common examples for multidimensional representations have been proposed by several authors including Chernoff's faces [23] or Fienberg's star plots [24]. Depending on the raw data and the case of application, a combination of various techniques is conceivable. For a detailed overview of visualization techniques please refer to [25] or [26]. In this work, we chose parallel coordinates due to their easy to understand and intuitive presentation.

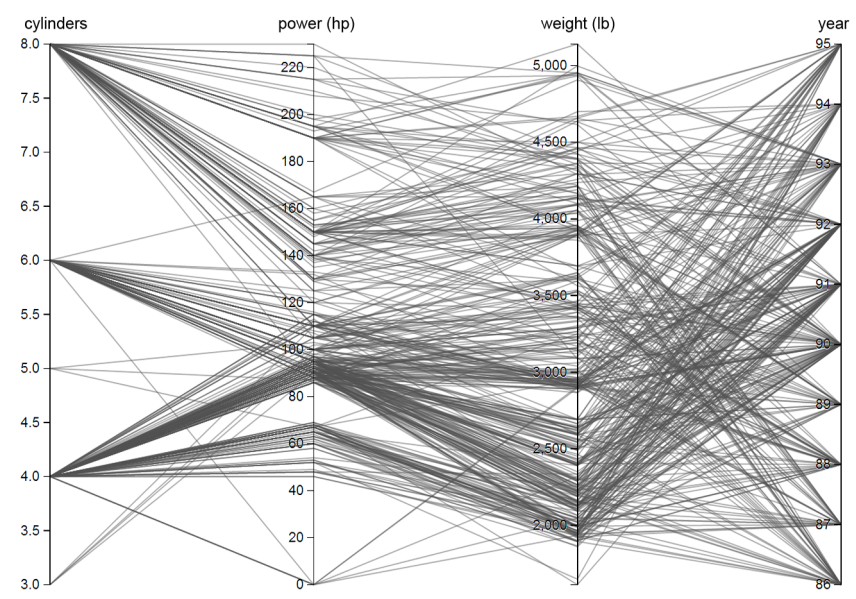

Figure 2. Parallel coordinates visualization of car data

Parallel coordinates is a well-known and often used visualization technique for illustrating multi-dimensional data sets. To project $n$ dimensions to a 2-dimensional visualization space, $n$ axes are laid out in parallel side-by-side. A point in the n-dimensional space is then represented as a polyline with vertices on the parallel axes [27]. Figure 2 shows an exemplary car data set ${ }^{1}$ visualized with parallel coordinates. Here, the attributes "cylinders", "power", "weight" and "year" are depicted. A limitation of this representation is that one axis can have at most two neighboring axes. Therefore, correlations may not be visible at a first glance. However, since the axes do not have a natural order,

\footnotetext{
${ }^{1}$ Obtained from: http://lib.stat.cmu.edu/datasets/
}

they could be reordered in various arrangements. A more effective way to cope with this limitation is user interaction.

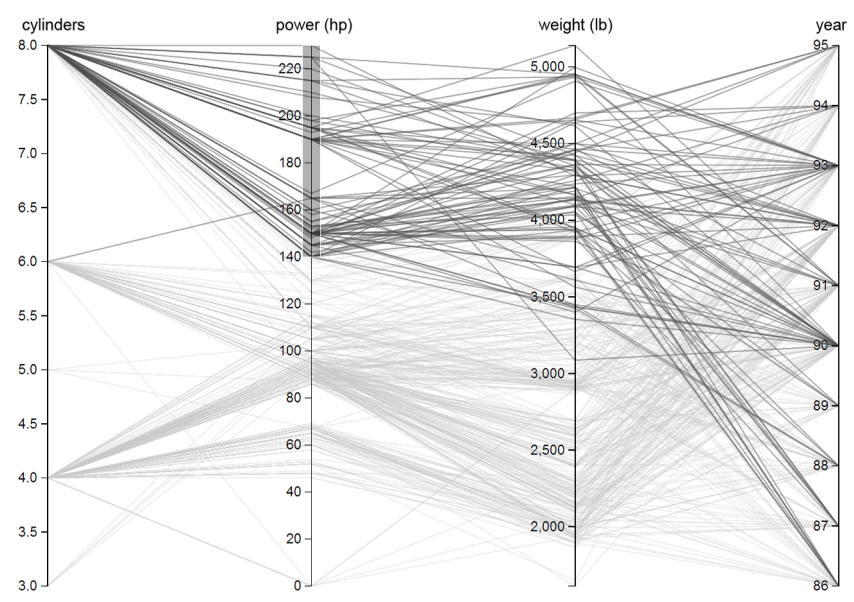

Figure 3. Brushed parallel coordinates visualization of car data. Cars with a power higher than $140 \mathrm{hp}$ are highlighted.

Used as an interactive tool, the exploration of correlations between single dimensions can be effectively supported through brushing. Brushing is a powerful technique that allows to focus on a subset of the data visualized by highlighting of the subset [28]. Thereto, particular segments of one or several axes have to be marked. In this way, correlations can be clearly revealed. Figure 3 depicts parallel coordinates with the same data set as before except that those cars that have a power of more than $140 \mathrm{hp}$ are emphasized. Obviously there is a strong correlation between horsepower and number of cylinders.

This generic conceptual design can be applied in various application areas as long as the two steps described are accurately performed. To demonstrate our visualizationbased approach, we implemented a prototype by the example of an eCommerce data set. This prototype may be applied in different eCommerce settings, since the context attributes identified are alike for most scenarios.

\section{B. Implementation using the example of an eBay dataset}

For reasons of demonstration, we collected a real life data set from eBay Germany containing feedback profiles of various users. As expounded in our conceptual design, the first step to carry out is to derive a model from the raw data provided. We analyzed the data set and identified the following attributes for our context set $C$ :

- Rating $\left(c_{1}\right)$ : The rating is the general seller evaluation conducted by the buyer after a transaction was carried out. It can take values of the discrete numerical interval $C_{1}=\{-1,0,1\}$, where -1 stands for a negative, 0 for a neutral and 1 for a positive rating.

- Time $\left(c_{2}\right)$ : The time describes the point of time a rating was created. Time is one essential context attribute, 


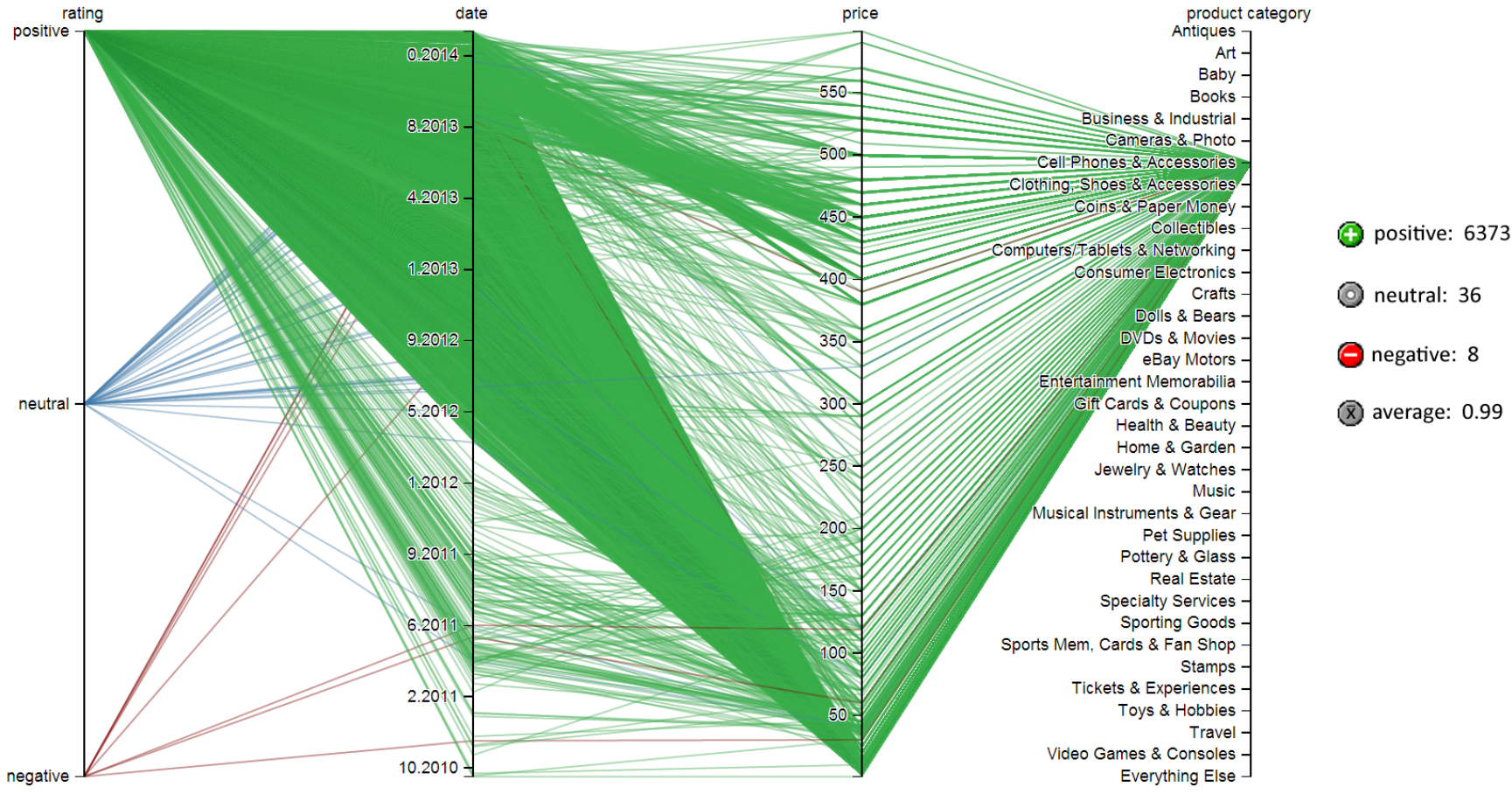

Figure 4. Exemplary eBay feedback profile of a merchant selling mobile devices. Green polylines depict positive, blue polylines neutral and red polylines depict negative ratings.

since old feedback might not be as relevant for reputation scoring as new referrals [29]. Time is a continuous interval bounded from above by the current timestamp.

- Price $\left(c_{3}\right)$ : The price describes the purchase price a transaction was coming off. As it is not necessarily bounded from above, the price takes values of the continuous interval $C_{3}=[0, \infty)$. The price (value) is the main example for the value imbalance problem, in which a seller could build high reputation selling cheap products while cheating on the expensive ones.

- Product category $\left(c_{4}\right)$ : The product type denotes the category a sold product was classified in. It is an interesting context attribute since a seller who sells good chewing gum might not necessarily sell high quality computers. The product hierarchy on ebay comprises 35 first level categories. Thus $c_{4}$ takes nominal values of a discrete set $C_{4}=\{$ "Antiques","Art", ... ,"Video Games \& Consoles", "Everything else"\}. For the sake of simplicity, we assume that each product is distinctly assigned to one category.

This results in our final referral space $S$ :

$$
S:=C_{1} \times C_{2} \times C_{3} \times C_{4}
$$

Every referral (tupel) of an ebay feedback profile denotes one point in the 4-dimensional referral space $S$ where each dimension matches one context attribute $c_{i}$. An example would be $s_{1}=(1$, '2013-12-26 06:35:40', 10.20, 'Travel') for a positive rating given on December the 26th in 2013 The transaction involved a product of the category Travel' that costed $10.20 €$.

To map this model to a 2-dimensional visualization space we implemented software prototype within a three-tier client server architecture. On the data layer we use a MySQLdatabase which contains all eBay profile data collected. The logic layer is implemented employing an Apache webserver. On the presentation layer, we created the parallel coordinates visualization using HTML5, CSS, SVG and JavaScript with the d3.js-package ${ }^{2}$. D3 (data-driven-documents) is a JavaScript library that allows to manipulate documents based on data. Applying d3, data are bound to the browser's DOM enabling the user to instantly interact and manipulate the visualization.

Figure 4 shows an exemplary parallel coordinates visualization depicting a feedback profile of an eBay merchant only selling mobile devices. Polylines denoting a positive rating are colored green, while neutral and negative rating are colored blue and red. On the right side, the values for positive, neutral and negative ratings as well as the average give an overview of the (absolute) numerical values. The visualization makes obvious that the merchant sold products within a range of $€ 1$ to nearly $€ 600$. The largest share of the transactions involved products cheaper than $€ 50$.

\footnotetext{
${ }^{2} \mathrm{http}: / / \mathrm{d} 3 \mathrm{js}$. org
} 
Furthermore, we see that the seller started to become a "powerseller" in spring of 2012. All transactions regard products of the category "Cell Phones \& Accessories". To reveal correlations between single attributes, the interaction techniques brushing can be applied. Figure 5 illustrates the same feedback profile with all polylines denoting a negative rating being emphasized. There is obviously no correlation between a negative ratings and another context attribute. Only sporadic negative ratings indicate a good overall quality of service. Further analyses include, for instance, an evaluation of specific product categories. If a buyer wants to make a bid for a video game, it would be interesting for him how the seller was rated for products of this category in particular. Actors interested in high price products should, moreover, mark the upper part of the price scale to reveal a possible correlation between high prices and negative ratings.

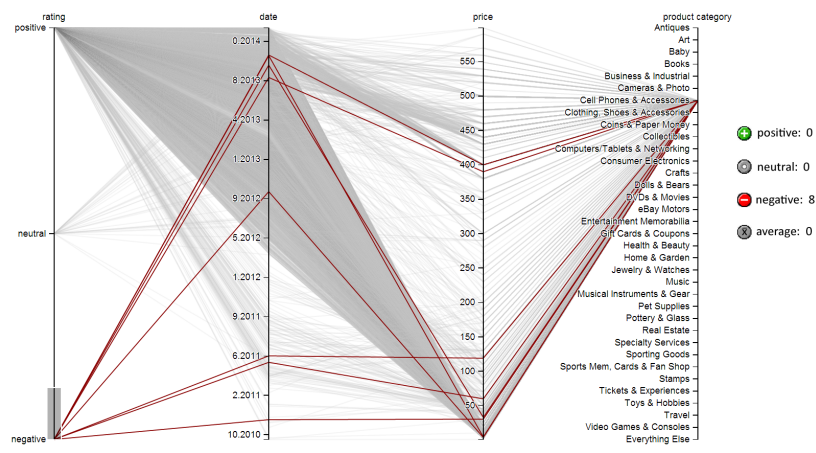

Figure 5. Brushing of all negative ratings reveals no correlation between the rating and other context attributes.

Overall, this presentation technique seems quite intuitive and easy to use. All information important for a surface impression can be obtain at a first glance. To carry out more detailed analyses, brushing offers an effective and powerful way to highlight correlations. To give an impression of how our prototype proves itself in practice we subsequently conduct an empirical study.

\section{EMPIRICAL STUDY}

In this section, two empirical cases are presented to demonstrate how value is added through the visual exploration of reputation profiles. We collected real-life data from 10 feedback profiles of malicious sellers on eBay Germany. Malicious sellers were identified through buyer complaints in eBay's community boards ${ }^{3}$. The community boards allow users to ask for help if there are any questions or problems regarding their transactions. Thereby, most of the users directly refer to the feedback profile of the seller in focus. At the time we analyzed the profiles, the complaining users have already been betrayed. Here, we show how

\footnotetext{
${ }^{3}$ http://community.ebay.de/
}

the deception could have been prevented by evaluating the referrals in advance using our visual representation.

To perform an exemplary analysis, we chose two of those profiles that allow to gain interesting insights.

\section{A. Case 1: Decreasing quality of service}

In this example we introduce a feedback profile of an eBay seller primarily specializing in selling autograph cards of soccer players. The user has been selling autograph cards on eBay for more than five years. The prices of the transactions carried out range from $€ 1$ to a maximum of $€$ 62.90. Figure 6 depicts the seller's profile summary as usually shown on eBay. Here, we translated the original text from German and blurred the seller's username as well as the accession date.

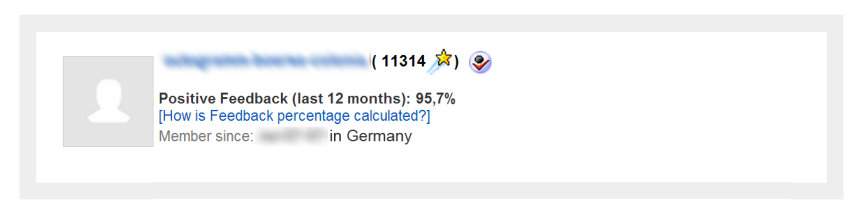

Figure 6. Seller profile (translated from German)

With over eleven thousand positive reviews, this seller seems to be reliable at a first glance (aggregate value 11314 indicates that there are at least 11314 positive ratings). However, a share of only $95.7 \%$ positive referrals in the last 12 months makes one become suspicious. Using our visual representation of the feedback profile it becomes clear that there is a strong correlation between negative ratings and time as depicted on Figure 7. Obviously the quality of service has been strongly decreasing in the recent weeks. Nearly all negative ratings were given in the last month this seller was active. At this time, it was no longer advisable to buy from this merchant.

Admittedly, the fact that the quality of service is currently quite bad could also have been found out easily through reading recent reviews in the seller's feedback profile. However, it would have required much more effort to get an overview of the full history and realize that bad behavior is a recent state. Only the integration of all referrals to one visualization allows to gain an overall picture. More sophisticated insights could be gained in the second case.

\section{B. Case 2: Value imbalance problem}

Since the value imbalance problem has already been known for quite a time, it was even more astonishing for us that we came across a seller benefiting from this issue very soon. As mentioned above, actors that profit from the value imbalance problem build high reputation by selling cheap products while cheating on the expensive ones. The seller's feedback profile (summary on Figure 8) listed 1139 positive, 1 neutral and 55 negative reviews resulting in an average of 


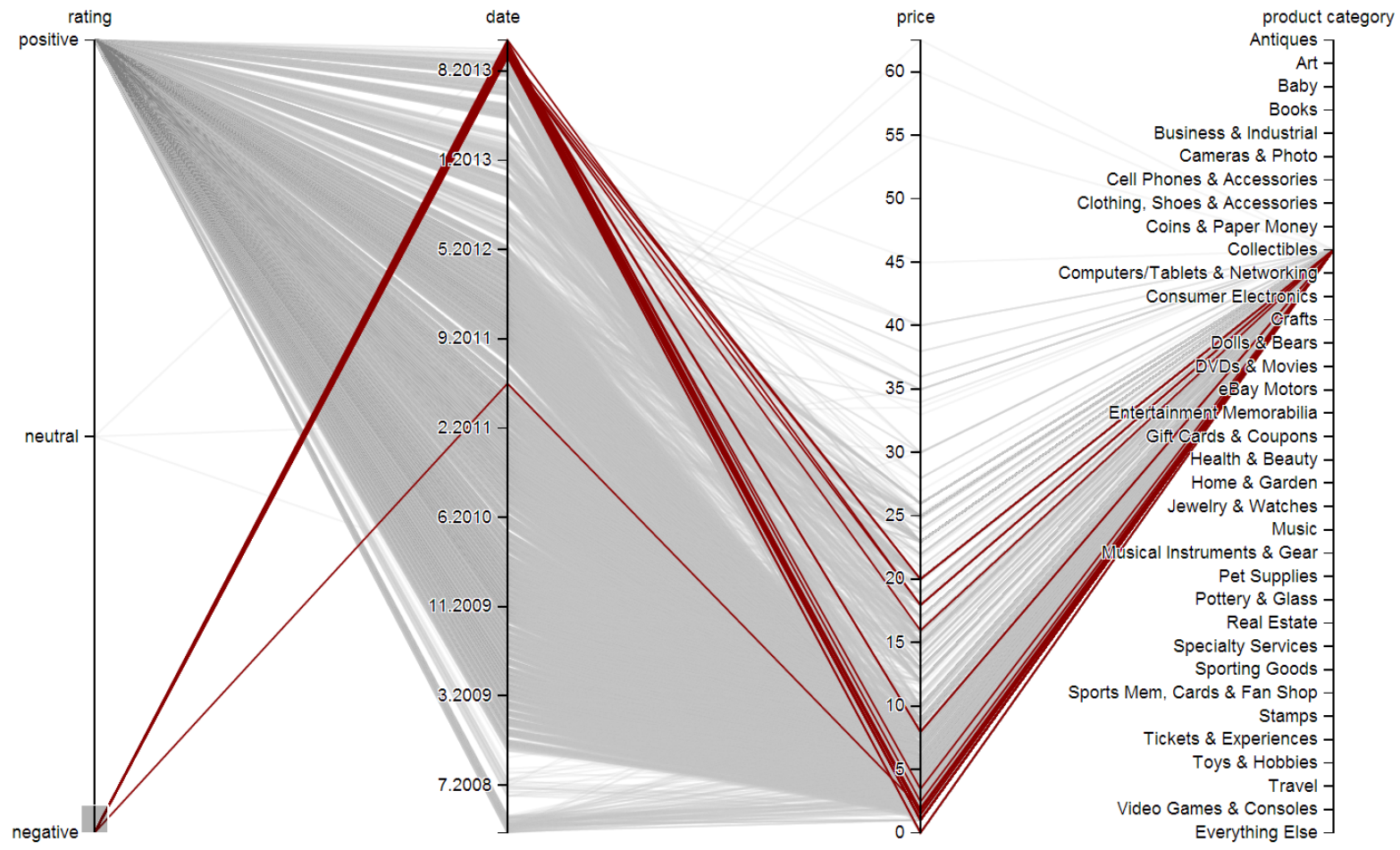

Figure 7. Seller profile with brushed polylines denoting negative rating. The upper end of the date scale show ratings created very recently.

95,3\% positive ratings in the last 12 months. At the time it was analyzed, it had an age of 5 months only. We came across this profile through a thread in eBay's community boards. A buyer complained that he had not yet received the mobile phone he payed one month before.

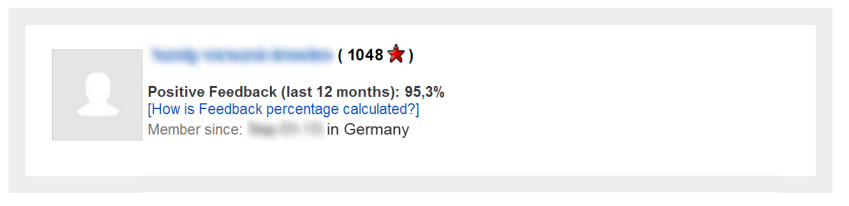

Figure 8. Seller profile 2 (translated from German)

Evaluating the reviews, we found that there were several but sporadic negative ratings. Each of these was nestled in many positive referrals, though. On the first page of the review list, we could find nothing but positive ratings of distinct users. This might be the reason why the malicious seller was still undetected. The visual exploration using parallel coordinates and the brushing of negative ratings, however, revealed that the merchant built up good reputation selling low-priced items while cheating on the expensive ones. All negative ratings involved products of a price higher than $€ 260$. Figure 9 depicts the profile in a parallel coordinates visualization with all negative ratings being highlighted (red).

Summing up the prices of those transactions having been rated negatively results in a total amount of more than $€ 25.000$. The fact that over 50 persons fell into the malicious seller's trap gives a lead that eBay's current seller profiles are not capable of revealing such attacks. A visual representation, however, would be an easy and very intuitive extension to prevent deception. Applying a contextsensitive reputation metric instead, it would also indicate that the seller is less trustworthy dealing with high prices. The numerical value, though, could not have delineated the overall coherence and hence reach transparency.

\section{Contributions And Future Work}

As demonstrated above, we could show that an interactive visual representation of transaction context of reputation data can notably increase transparency by giving an overview of all referrals in one picture and letting the users explore the data sets. If users gain insights by themselves, they will logically have a higher level of trust toward the reputation system since it does not only provide a pre-calculated reputation value but rather allow to evaluate the referrals and derive one's reputation. Our empirical studies furthermore illustrated how malicious profiles could easily be detected by the user. A visual representation, though, may not totally replace a numerical analysis. Thus, our visual approach could be used as an extension to make the computation process more transparent. Through representing all input data, replicability is achieved. 


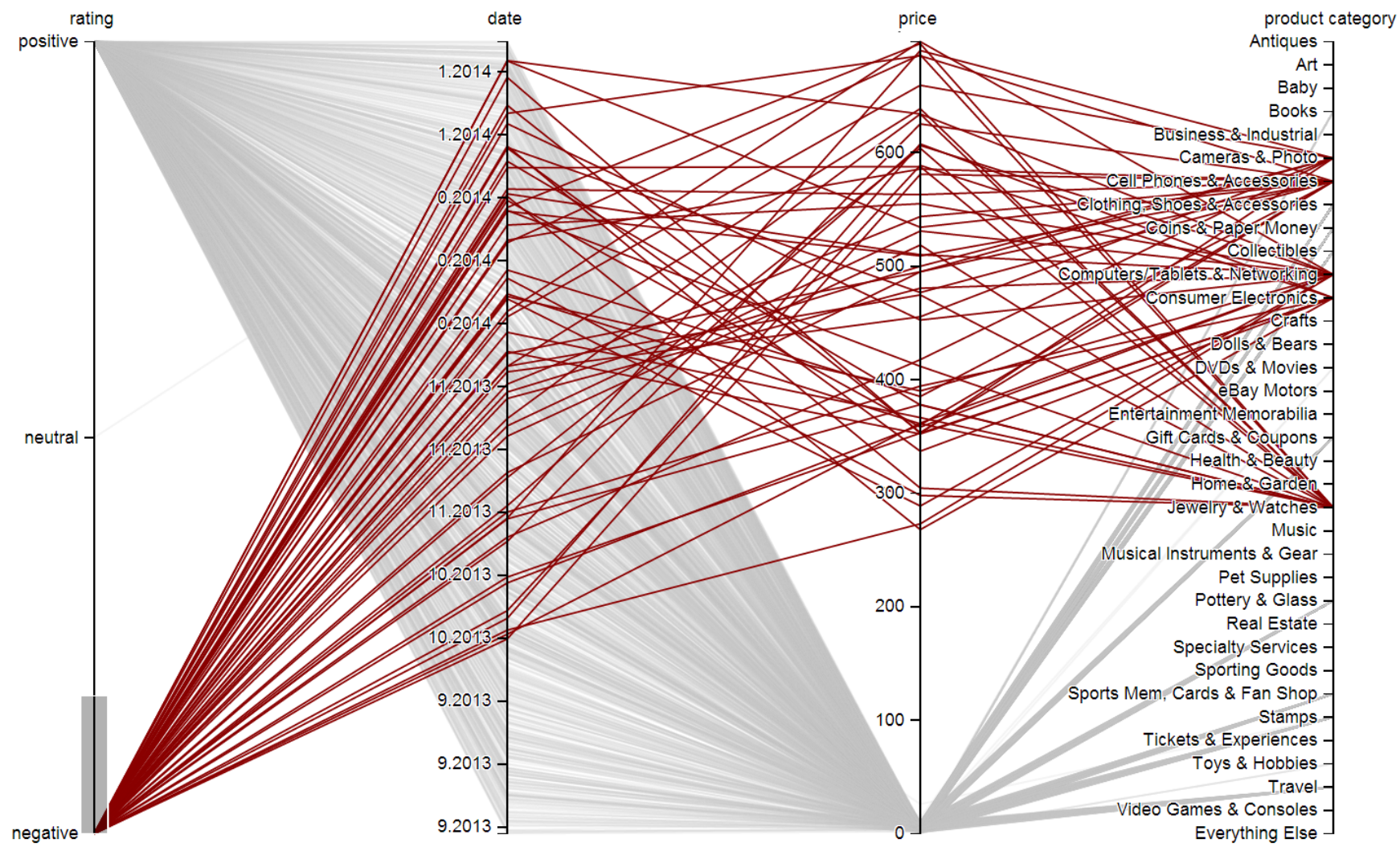

Figure 9. Seller profile with brushed polylines denoting negative rating strongly correlating with high prices.

However, there are still many unexplored questions that need to be answered. The following list gives an overview of those issues that will be topic for our future research:

- User study: Even though our empirical study depicted how the visual representation could be used for visual data exploration, it has not been proven if a average end-user could effectively work with our prototype in practice. Thus, a user study needs to be carried out in which the usability, effectiveness and applicability is evaluated. Thereby, the value added compared to other context-sensitive approaches needs to be clarified. Further questions regard user acceptance. We need to evaluate if the users want to be integrated in the computation process.

- Enhanced presentation: Parallel coordinates is only one visualization technique for illustration of multivariate data. Interesting future projects involve an evaluation of further visualization and interaction techniques as well as an integration to one reputation assessment cockpit. Here again, it needs to be clarified if the user might be "over-challenged" by multiple visualization and interaction techniques.

- Implementation in further application areas: We demonstrated how our approach can be implemented by the example of an eCommerce settings. That raises the question of what differences, challenges and benefits appear in other application areas. Interesting fields could be online collaborations or the rating of web service providers.

- Detection of attacks: The cases introduced in this work focused on malicious actors making use of deficient consideration of transaction context. Visualization and interaction techniques might also be expedient for detecting further attacks on reputation systems. A visual representation of the referral network, for instance, might help to detect sybil attacks. Thus, further research to identify potentially helpful techniques is necessary.

\section{CONCLUSION}

Visual analytics is a steadily growing field of research that is applied in multiple application areas. In this work, we were taking the first step to make use of visual analytics in trust and reputation systems. We introduced a generic conceptual design where the ratings are extended by multiple context attributes to form an n-dimensional referral space. This referral space is then mapped to a 2-dimensional visualization space and illustrated in a parallel coordinates visualization allowing an interactive exploration of data sets. We furthermore demonstrated how this generic mechanism can easily be implemented by the example of an eCommerce 
data set from eBay. The subsequent case studies could prove its usefulness in a practical setting.

Applying our novel approach, we enhance transparency by depicting all referrals as well as the context they were created in one integrated view. Thus, decisions and calculations made by the reputation system become replicable. We furthermore allow to involve the user in the evaluation process and let the user gain insights and draw conclusions by himself. As a consequence, we increase the users' trust in the reputation system.

Overall, this work can be seen as a starting point for further research in reputation evaluation through visual analytics. Since visual analytics is a wide field of research offering hundreds of visualization and interaction techniques, we see a great potential in the combination of both fields. In this work, we named several questions that need to be clarified in future research. The answers might improve and change the generic design of reputation systems in the long term.

\section{ACKNOWLEDGMENT}

The research leading to these results was supported by the Bavarian State Ministry of Education, Science and the Arts" as part of the FORSEC research association.

\section{REFERENCES}

[1] Y. Yao, S. Ruohomaa, and F. Xu, "Addressing Common Vulnerabilities of Reputation Systems for Electronic Commerce," Journal of theoretical and applied electronic commerce research, vol. 7, no. 1, pp. 1-20, 2012.

[2] M. Rehak, M. Gregor, M. Pechoucek, and J. Bradshaw, "Representing Context for Multiagent Trust Modeling," in Proceedings of the IEEE/WIC/ACM International Conference on Intelligent Agent Technology, ser. IAT '06, Hongkong and China, 2006, pp. 737-746.

[3] R. Kerr and R. Cohen, "Modeling Trust Using Transactional, Numerical Units," in Proceedings of the 2006 International Conference on Privacy, Security and Trust: Bridge the Gap Between PST Technologies and Business Services, ser. PST '06. New York and NY and USA: ACM, 2006, pp. 21:1-21:11. [Online]. Available: http://doi.acm.org/10.1145/ 1501434.1501460

[4] H. Zhang, Y. Wang, and X. Zhang, "A trust vector approach to transaction context-aware trust evaluation in e-commerce and e-service environments," in Proceedings of the 5th IEEE International Conference on Service-Oriented Computing and Applications, Taipei and Taiwan, 2012, vol. SOCA, pp. 1-8.

[5] Stephan Hammer, Rolf Kiefhaber, Matthias Redlin, Elisabeth Andre, and Theo Ungerer, "A User-Centric Study Of Reputation Metrics in Online Communities," in 3rd Workshop on Trust, Reputation and User Modeling (TRUM'13), Rome and Italy, 2013.

[6] Alan R. Hevner, Salvatore T. March, Jinsoo Park, and Sudha Ram, "Design Science in Information Systems Research," MIS Quarterly, vol. 28, no. 1, pp. 75-105, 2004.
[7] K. Peffers, T. Tuunanen, M. Rothenberger, and S. Chatterjee, "A Design Science Research Methodology for Information Systems Research," J. Manage. Inf. Syst., vol. 24, no. 3, pp. 45-77, 2007. [Online]. Available: http://dx.doi.org/10.2753/ MIS0742-1222240302

[8] D. H. McKnight and N. L. Chervany, "The meanings of trust," Technical Report MISRC Working Paper Series 96-04, University of Minnesota, Management Information Systems Reseach Center, 1996.

[9] D. Gambetta, "Can We Trust Trust?" in Trust: Making and Breaking Cooperative Relations, D. Gambetta, Ed. Oxford: Basil Blackwell, 1988, pp. 213-237.

[10] C. Castelfranchi and Y.-H. Tan, Eds., Trust and deception in virtual societies. Dordrecht and Boston: Kluwer Academic Publishers, 2001.

[11] D. Artz and Y. Gil, "A survey of trust in computer science and the Semantic Web," Web Semantics, vol. 5, no. 2, pp. 58-71, 2007.

[12] A. Jøsang, R. Ismail, and C. Boyd, "A survey of trust and reputation systems for online service provision," Decision Support Systems, vol. 43, no. 2, pp. 618-644, 2007.

[13] A. K. Dey, "Understanding and Using Context," Personal and Ubiquitous Computing, vol. 5, no. 1, pp. 4-7, 2001. [Online]. Available: http://dx.doi.org/10.1007/s007790170019

[14] G. Abowd, A. Dey, P. Brown, N. Davies, M. Smith, and P. Steggles, "Towards a Better Understanding of Context and Context-Awareness," in Handheld and Ubiquitous Computing, ser. Lecture notes in computer science, H.-W. Gellersen, Ed. Springer Berlin Heidelberg, 1999, vol. 1707, pp. 304307.

[15] G. Adomavicius and A. Tuzhilin, "Toward the next generation of recommender systems: a survey of the state-of-the-art and possible extensions," Knowledge and Data Engineering, IEEE Transactions on, vol. 17, no. 6, pp. 734-749, 2005.

[16] S. K. Mostefaoui and B. Hirsbrunner, "Context aware service provisioning," in Pervasive Services, 2004. ICPS 2004. Proceedings. The IEEE/ACS International Conference on, 2004, pp. 71-80.

[17] J. Sabater and C. Sierra, "REGRET: Reputation in Gregarious Societies," in Proceedings of the Fifth International Conference on Autonomous Agents, ser. AGENTS '01. New York and NY and USA: ACM, 2001, pp. 194-195. [Online]. Available: http://doi.acm.org/10.1145/375735.376110

[18] M. G. Uddin, M. Zulkernine, and S. I. Ahamed, "CAT: A Context-aware Trust Model for Open and Dynamic Systems," in Proceedings of the 2008 ACM Symposium on Applied Computing, ser. SAC '08. New York and NY and USA: ACM, 2008, pp. 2024-2029. [Online]. Available: http://doi.acm.org/10.1145/1363686.1364176

[19] Z. Malik and A. Bouguettaya, "RATEWeb: Reputation Assessment for Trust Establishment Among Web Services," The VLDB Journal, vol. 18, no. 4, pp. 885-911, 2009. [Online]. Available: http://dx.doi.org/10.1007/s00778-009-0138-1 
[20] D. A. Keim, J. Kohlhammer, G. Ellis, and F. Mansmann, Mastering the Information Age - Solving Problems with Visual Analytics. Eurographics Association, 2010. [Online]. Available: http://books.google.de/books?id=vdv5wZM8ioIC

[21] D. Keim, G. Andrienko, J.-D. Fekete, C. Görg, J. Kohlhammer, and G. Melançon, "Visual Analytics: Definition, Process, and Challenges," in Information Visualization, ser. Lecture notes in computer science, A. Kerren, J. Stasko, J.-D. Fekete, and C. North, Eds. Springer Berlin Heidelberg, 2008, vol. 4950, pp. 154-175.

[22] J. O'Donovan, B. Smyth, V. Evrim, and D. McLeod, "Extracting and Visualizing Trust Relationships from Online Auction Feedback Comments," in Proceedings of the 20th International Joint Conference on Artifical Intelligence, ser. IJCAI'07. San Francisco and CA and USA: Morgan Kaufmann Publishers Inc, 2007, pp. 28262831. [Online]. Available: http://dl.acm.org/citation.cfm?id= 1625275.1625730

[23] H. Chernoff, The use of faces to represent points in $n$ dimensional space graphically, ser. Technical report / Department of Statistics, Stanford University. Stanford and Calif: Dept. of Statistics Stanford Univ, 1971, vol. 71.

[24] S. E. Fienberg, Graphical methods in statistics, ser. Technical report / University of Minnesota (Minneapolis-St. Paul Campus). Dept. of Applied Statistics. Minneapolis: University of Minnesota Microfilm Service, 1981, vol. no. 304.

[25] W. Aigner, S. Miksch, H. Schumann, and C. Tominski, "Survey of Visualization Techniques," in Visualization of Time-Oriented Data, ser. Human-Computer Interaction Series. Springer London, 2011, pp. 147-254.

[26] C. D. Hansen and C. R. Johnson, The Visualization Handbook, ser. Referex Engineering. ButterworthHeinemann, 2005. [Online]. Available: http://books.google. de/books?id=ZFrlULckWdAC

[27] E. J. Wegman, Hyperdimensional data analysis using parallel coordinates, Fairfax and Va, 1987.

[28] H. Hauser, F. Ledermann, and H. Doleisch, "Angular Brushing of Extended Parallel Coordinates," in Proceedings of the IEEE Symposium on Information Visualization (InfoVis'02), ser. INFOVIS '02. Washington and DC and USA: IEEE Computer Society, 2002, pp. 127-. [Online]. Available: http://dl.acm.org/citation.cfm?id=857191.857742

[29] M. Tavakolifard and K. C. Almeroth, "A Taxonomy to Express Open Challenges in Trust and Reputation Systems," Journal of Communications, vol. 7, no. 7, pp. 538-551, 2012. 